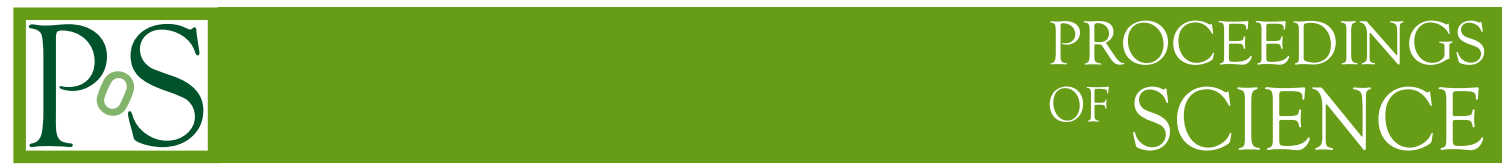

\title{
Flavour physics from lattice QCD
}

\author{
Elvira Gámiz*† \\ CAFPE and Depto. de Física Teórica y del Cosmos, Universidad de Granada, E-18002, \\ Granada, Spain \\ E-mail: megamiz@ugr.es
}

I review recent results and prospects for improvement in calculations of hadronic matrix elements relevant to quark flavour phenomenology. I will focus on key quantities for CKM unitarity triangle analyses and for the study of discrepancies between experiment and SM predictions for some flavour observables.

Xth Quark Confinement and the Hadron Spectrum,

October 8-12, 2012

TUM Campus Garching, Munich, Germany

\footnotetext{
* Speaker.

$\dagger$ The author's work is supported in part by the MICINN under Grants FPA2010-16696 and FPA2006-05294, and Ramón y Cajal program; by Junta de Andalucía under Grants FQM-101, FQM-330, and FQM-6552; and by European Commission under Grant No. PCIG10-GA-2011-303781.
} 


\section{Introduction}

The flavour physics program plays a dominant role in testing the Standard Model (SM) and searching for New Physics (NP), providing information which is complementary to direct searches in colliders. NP effects could be unveiled through the observation of deviations from the SM via high-precision measurements of low-energy observables in high-luminosity experiments. Indeed, there are several measurements of flavour observables for which there is a $2-3 \sigma$ difference from SM predictions. These include $\sin (2 \beta)$, the like-sign dimuon charge asymmetry, $B$ leptonic decays, unitarity triangle (UT) fits, and, more recently, the ratios of branching fractions of $B$ semileptonic decays to a $D$ and a $\tau$ over the corresponding decays to a $l=e, \mu$.

In order to test the Cabibbo-Kobayashi-Maskawa (CKM) description of the experimentally measured CP-violating and flavour changing processes, and investigate the origin of the discrepancies mentioned above, we need a determination of the weak matrix elements involved in those processes with matching precision. In many cases, lattice QCD can provide those non-perturbative theoretical inputs from first principles and with errors at a few per-cent level. Accuracy in lattice calculations requires control over all the sources of systematic error. In particular, it is essential to take into account vacuum polarization effects in a realistic way, i.e., including up, down and strange sea quarks on the gauge configurations' generation. The up and down quarks are usually taken to be degenerate, so those simulations are referred to as $N_{f}=2+1$. Two lattice collaborations (FNAL/MILC and ETMC) are now generating configurations which also include the effects of charm quarks on the sea, $N_{f}=2+1+1$. The first preliminary results for flavour quantities on those configurations are starting to appear. In the next Sections I will discuss the latest results for non-perturbative quantities relevant for flavour studies from lattice QCD calculations with all sources of systematic error addressed.

\section{Light quarks matrix elements}

\section{1 $\left|V_{u s}\right|$ from $K$ leptonic and semileptonic decays}

The lattice calculation of pseudoscalar decay constants, together with experimental measurements of pseudoscalar leptonic decay widths, can be used to extract the value of the CKM matrix elements involved in the process. The decay constants are easy to calculate on the lattice with high precision. The accuracy achieved is even higher for ratios of decay constants, for example $f_{K} / f_{\pi}$, since many systematic uncertainties and statistical fluctuations cancel partially or completely between numerator and denominator. $f_{K} / f_{\pi}$ has been widely studied on the lattice and the precision has reached the subpercent level. The average of the published results with $N_{f}=2+1$ is [1] $f_{K} / f_{\pi}=1.1936 \pm 0.0053$. Agreement between different collaborations also provides a good check of lattice methodologies. The phenomenological interest of this quantity stems from the fact that it is related to the ratio $\left|V_{u s}\right| /\left|V_{u d}\right|$ and experimental measurements of $K$ and $\pi$ leptonic decay widths, so it can be used to extract a value for $\left|V_{u s}\right|$. Using the experimental average in [2], and the lattice average for $f_{K} / f_{\pi}$ above, we get $\left|V_{u s}\right|=0.2252(11)$.

Precise determinations of $\left|V_{u s}\right|$ provide stringent tests of first-row unitarity and give information about the scale of NP [3]. This CKM parameter can also be extracted from experimental data on $K$ semileptonic decay rates, given the value of the vector form factor at zero momentum transfer, 
$f_{+}^{K \pi}(0)$. The parameter $f_{+}^{K \pi}(0)$ is also one of the inputs used in UT analyses. The only unquenched lattice calculations available for this form factor ${ }^{1}$ are the ones by the RBC/UKQCD and ETMC collaborations [5]. The average of the two calculations is [1] $f_{+}^{K \pi}(0)=0.9584(44)$, which gives $\left|V_{u s}\right|=0.2257(11)$, in perfect agreement with the value extracted from leptonic decays and also in agreement with unitarity.

\section{$2.2 K^{0}-\bar{K}^{0}$ mixing}

The information coming from neutral Kaon mixing provides one of the most stringent constraints in UT analyses. Until a few years ago, the limiting factor to exploit that constraint was the uncertainty in the calculation of the hadronic matrix element which encodes the non-perturbative physics of the process, parametrized by the bag parameter $B_{K}$. This is no longer true thanks to several $N_{f}=2+1$ lattice QCD calculations, four of them in the last year [6], which have reduced the error of $B_{K}$ under $2 \%$. The average of all $N_{f}=2+1$ calculations is [1] $\hat{B}_{K}=0.7643(97)$. This year we have also had the first unquenched $\left(N_{f}=2\right)$ calculation of the matrix elements of the four-fermion operators contributing to $K^{0}-\bar{K}^{0}$ in extensions of the SM [7].

\section{Heavy quark phenomenology}

\subsection{Heavy-light decay constants}

The decay constant of the $D_{s}$ meson, $f_{D_{s}}$, has generated a vivid interest in both experimentalists and lattice QCD phenomenologists in the last years, especially since 2007, when an important reduction in the errors of the lattice calculation of $f_{D_{s}}$ made possible to observe a $3.8 \sigma$ disagreement between the lattice and experimental averages. Later improvements in both the theoretical and the experimental sides, however, shift both numbers and reduced the difference. Nevertheless, the most precise lattice calculations, including those in a preliminary stage, tend to give smaller values of $f_{D_{s}}$ than experiment. In particular, two $N_{f}=2+1+1$ preliminary results from the ETMC and the FNAL/MILC collaborations, are around $(245-250) \mathrm{MeV}$ [8]. The lattice averages, which only include the $N_{f}=2+1$ calculations already complete, are $[1,9,10]$

$$
f_{D}=209.2(3.9) \mathrm{MeV}, f_{D_{s}}=248.6(3.0) \mathrm{MeV} \text {. }
$$

The value of $f_{D}$ agrees with experiment [11], $f_{D}=206.7 \pm 8.9$, while $f_{D_{s}}$ is around $2 \sigma$ lower than the experimental average [12], $f_{D_{s}}=(260.0 \pm 5.4) \mathrm{MeV}$. The preliminary BESIII result in [13], $f_{D}=(203.9 \pm 6.0) \mathrm{MeV}$, agrees well with the CLEO result and the lattice average.

UT fits are very sensitive to $f_{B}$ and different processes with potential to show up NP effects depend on $f_{B}$ or $f_{B_{s}}$, so any improvement in the decay constants calculations as well as in the understanding of the $\left|V_{u b}\right|^{\text {exc. }} /\left|V_{u b}\right|^{\text {inc. }}$ disagreement is very important. There have been three lattice $N_{f}=2+1$ calculations of this parameter in the last two years by the HPQCD and FNAL/MILC [14] collaborations, which have reduced the error at the $2.5 \%$ level. The average values of $f_{B}$ and $f_{B_{s}}$

\footnotetext{
${ }^{1}$ After this conference, the FNAL/MILC collaboration has provide another result [4], compatible with those by the $\mathrm{RBC/UKQCD}$ and ETMC collaborations, but with a larger central value and smaller errors. The FNAL/MILC result implies $\left|V_{u s}\right|=0.2238 \pm 0.0009 \pm 0.0005$.
} 
from these three calculations are [1]

$$
f_{B}=190.6(4.7) \mathrm{MeV}, f_{B_{s}}=227.6(5.0) \mathrm{MeV} .
$$

The direct comparison of the results in Eq. (3.2) with experimental measurements of the $B$ leptonic decay width is problematic due to the need of the value of the CKM matrix element $\left|V_{u b}\right|$ (whose inclusive and exclusive determinations disagree at the $3 \sigma$ level) and the $\sim 2 \sigma$ disagreement of BaBar [15] and Belle's [16] measurements. Nevertheless, Belle new result seems to alleviate the tension between theory and experiment previously observed.

\section{2 $B$ semileptonic decays}

There exist $\sim 2-3 \sigma$ tensions between the inclusive and the exclusive determinations of both $\left|V_{u b}\right|$ and $\left|V_{c b}\right|$. In addition to experimental measurements on, for example, $B \rightarrow \pi l v$ and $B \rightarrow D\left(D^{*}\right) l v$, respectively, the exclusive determination of those CKM elements need as input form factors that can be calculated with high precision using lattice QCD techniques. There have not been new lattice QCD calculations of the form factors describing the $B \rightarrow \pi l v$ decay since 2008 [17], although several analyses are in progress. Using the lattice results in Ref. [17] and the latest experimental data [18], $\left|V_{u b}\right|_{\text {exclusive }}=(3.23 \pm 0.30) \cdot 10^{-3}$.

For the exclusive determination of $\left|V_{c b}\right|$, the state-of-the art calculation of the relevant form factors is the FNAL/MILC analysis in [19], which studies the decay $B \rightarrow D^{*} l v$ at zero recoil. The updated result is $\left|V_{c b}\right|=(39.7 \pm 0.7 \pm 0.7) \cdot 10^{-3}$, where the first error is experimental and the second one the uncertainty in the lattice calculation of the form factors. The FNAL/MILC collaboration is doing an extensive study of both $B \rightarrow D^{*} l v$ and $B \rightarrow D l v$ decays at zero and nonzero recoil [20], providing two independent modes for the extraction of $\left|V_{c b}\right|$. This study will also provide checks of the shape of the form factors, in addition to $\left|V_{c b}\right|$.

The FNAL/MILC collaboration recently analyzed a subset of their $B \rightarrow D l v$ data to calculate the ratio of branching fractions $R(D)=\mathscr{B} r(B \rightarrow D \tau v) / \mathscr{B} r(B \rightarrow D l v)=0.316(14)$ [21]. Their value is $\sim 1.7 \sigma$ smaller than the recent experimental measurement by the BaBar collaboration [22]. They found that the value of the ratio is very sensitive to differences in the scalar form factor, so one should be cautious in using indirect estimates of the form factors to constrain NP models in other decay channels such as $B \rightarrow D^{*} \tau \nu$ [21]. Given the present tensions, not only for $R(D)$ but for $R\left(D^{*}\right)$, and the possible indications of NP that could be extracted from a combined analysis of both set of decays, unquenched lattice QCD calculations of those two ratios should be a priority. Current experimental measurements of these ratios are statistics-limited, so Belle II should significantly reduce the errors of those measurements. The Cambridge group and the FNAL/MILC collaboration have also presented preliminary results for the different form factors describing the rare decays $B \rightarrow K\left(K^{*}\right) l^{+} l^{-}[23,24]$.

\section{3 $D$ semileptonic decays}

The semileptonic modes $D \rightarrow K l v$ and $D \rightarrow \pi l v$ allow us not only to extract the values of the CKM matrix elements $\left|V_{c s(d)}\right|$, but to test lattice QCD techniques and methodology by comparing the shape of the corresponding form factors, $f_{+}^{D K(\pi)}\left(q^{2}\right)$, with experimental data. The method developed by the HPQCD collaboration that employs a Ward identity to relate the matrix element 


\begin{tabular}{cccc}
\hline \hline & HPQCD & FNAL/MILC & RBC/UKQCD \\
\hline$\xi$ & $1.258(33)$ & $1.27(6)$ & $1.13(12)$ \\
\hline$B_{B_{s}} / B_{B_{d}}$ & $1.05(7)$ & $1.06(11)$ & - \\
HPQCD: $f_{B_{s}} \sqrt{\hat{B}_{B_{s}}}=266(6)(17) \mathrm{MeV}$, & $\hat{B}_{B_{s}}=1.33(6)$ \\
HPQCD: $f_{B_{d}} \sqrt{\hat{B}_{B_{d}}}=216(9)(13) \mathrm{MeV}$, & $\hat{B}_{B_{d}}=1.26(11)$ \\
\hline \hline
\end{tabular}

Table 1: $B$-meson mixing parameters. $\xi$ is defined as the ratio of the parameters in the second and third rows. In the case where there are two errors, the first one is statistical and the second one systematic.

of a vector current to that of the corresponding scalar current, and the use of highly improved lattice discretizations to treat the charm quarks relativistically, have allowed a reduction of the errors in the lattice calculation of the form factors $f_{+}^{D \rightarrow \pi}(0)$ and $f_{+}^{D \rightarrow K}(0)$ from around a $10 \%$ to a $5 \%$ and a $2.5 \%$ respectively. The average of the results in $[25,26]$ by the HPQCD and FNAL/MILC collaborations are

$$
\left|V_{c s}\right|=0.961(11)(24),\left|V_{c d}\right|=0.225(6)(10),
$$

where the first error is from experiment and the second one is the lattice error in $f_{+}^{D \rightarrow K(\pi)}(0)$. This is compatible with unitarity $\left(\left|V_{c s}\right|=0.97345(16)\right.$ and $\left.\left|V_{c d}\right|=0.2252(7)\right)$, and it is competitive with the determination of $\left|V_{c d}\right|$ from neutrino scattering, $\left|V_{c d}\right|=0.230(11)$ [27].

There are several on-going projects that study the dependence of the form factors on the momentum transfer, showing a good description of the experimentally measured shape. HPQCD has quoted a preliminary number extracted from a global fit to Belle, BaBar, and CLEO data, of $\left|V_{c s}\right|=0.965(14)[28]$.

\subsection{Neutral $B$-meson mixing}

It has been argued that differences observed between measurements of some flavor observables and the corresponding Standard Model (SM) predictions may be due to beyond the SM (BSM) physics affecting the neutral $B$-meson mixing processes [29, 30]. Although the most recent analysis seem to indicate that there are not large BSM contribution to neutral $B$-meson mixing [31], the future will bring new twists, and precise calculations of the non-perturbative inputs parametrizing the mixing in the SM and beyond are necessary for a thorough understanding of quark flavor physics. The current status of $N_{f}=2+1$ lattice calculations of the non-perturbative quantities parametrizing the mass differences between the heavy and the light mass eigenstates in both the $B_{d}^{0}$ and $B_{s}^{0}$ systems, as well as the $\mathrm{SU}(3)$ breaking ratio $\xi$, is summarized in Tab. 1. The HPQCD [32] and FNAL/MILC [33] collaborations use the same light quark formulation, but a different description for the $b$ quarks. In the exploratory study by the RBC/UKQCD collaboration in the table heavy quarks are static [34]. The average of the results in Tab. 1 for $\xi$ gives the value $\xi=1.251 \pm 0.032$.

Beyond the SM the mixing parameters can have contributions from $\Delta B=2$ four-fermion operators which do not contribute in the SM. The study of these contributions can provide very useful constraints on the allowed BSM theories. There is not a final unquenched lattice calculation of the matrix elements of all the operators in the $\Delta B=2$ effective hamiltonian, but FNAL/MILC and ETMC have presented preliminary results for the complete basis in $[35,36]$. Those preliminary 
results include the matrix elements needed for the determination of the decay width differences, $\Delta \Gamma_{d, s}$, for which there is not currently an unquenched calculation in the continuum limit.

The authors of Ref. [37] suggested that the branching fractions of the rare decays $B_{q} \rightarrow \mu^{+} \mu^{-}$ (for $q=s, d$ ) could be determined from the experimental measurement of the mass difference in the neutral $B_{q}$-meson system, $\Delta M_{q}$, and the lattice calculation of the bag parameter $\hat{B}_{B_{q}}$ since

$$
\mathscr{B} r\left(B_{q} \rightarrow \mu^{+} \mu^{-}\right) / \Delta M_{q}=[\text { knownfactors }] / \hat{B}_{q}
$$

In order to compare experimental measurements and the theory predictions for the decay rate of $B_{s}^{0}$, one should include the effects of a non-vanishing $\Delta \Gamma_{s}$ [38]. This can be done in the SM by rescaling the theory prediction by $1 /\left(1-y_{s}\right)$, where $y_{s} \equiv \tau_{B_{s}} \Delta \Gamma_{s} / 2$ [38]. Multiplying Eq. (3.4) by that factor for the $B_{s}^{0} \rightarrow \mu^{+} \mu^{-}$decay and using the HPQCD determination of the bag parameters $\hat{B}_{B_{s}}=1.33(6)$ and $\hat{B}_{B_{d}}=1.26(11)$ [32]; together with $\tau_{B_{s}}=1.497(15) p s, \tau_{B_{d}}=1.519(7) p s$ [27], and $\Delta \Gamma_{s}=0.116 \pm 0.019 \mathrm{ps}^{-1}$ [39], one gets

$$
\left.\mathscr{B} r\left(B_{s} \rightarrow \mu^{+} \mu^{-}\right)\right|_{y_{s}}=(3.65 \pm 0.20) \times 10^{-9}, \quad \mathscr{B} r\left(B_{d} \rightarrow \mu^{+} \mu^{-}\right)=(1.04 \pm 0.09) \times 10^{-10}(3.5)
$$

The direct calculation of these branching fractions have become competitive with the one in (3.5) [40] thanks to the recent improvements in the calculation of the $B$-meson decay constants on the lattice described in Sec. 3.1. Including the correction factor $1 /\left(1-y_{s}\right)$ for the $B_{s} \rightarrow \mu^{+} \mu^{-}$decay rate, and using the same inputs as in Ref. [40] except for $f_{B}$ and $f_{B_{s}}$, for which I use the averages described in Sec. 3.1, and $\tau_{B_{s}}$, which I take equal to its PDG 2012 value, $\tau_{B_{s}}=1.497(15) p s$, I get

$$
\left.\mathscr{B} r\left(B_{s} \rightarrow \mu^{+} \mu^{-}\right)\right|_{y_{s}}=(3.64 \pm 0.23) \cdot 10^{-9}, \quad \mathscr{B} r\left(B_{d} \rightarrow \mu^{+} \mu^{-}\right)=(1.07 \pm 0.10) \cdot 10^{-10} .
$$

The agreement between the two set of numbers in (3.5) and (3.6) is excellent, giving us confidence on the SM prediction for these branching fractions. This is very important since LHC is approaching the SM predictions, with the ïñ Arst evidence for one of these two processes [41], $\mathscr{B} r\left(B_{s} \rightarrow \mu^{+} \mu^{-}\right)=\left(3.2_{-1.2}^{+1.5}\right) \cdot 10^{-9}$, which is consistent with the SM prediction.

Another recent contribution of lattice QCD to the study of $B_{s} \rightarrow \mu^{+} \mu^{-}$rare decays has been the calculation by the FNAL/MILC collaboration of form-factors ratios between the semileptonic decays $\bar{B}^{0} \rightarrow D^{+} l^{-} \bar{v}$ and $\bar{B}_{s}^{0} \rightarrow D_{s}^{+} l^{-} \bar{v}$ [42]. These ratios are a key theoretical input in a new strategy to determine the fragmentation fractions of neutral $B$ decays, which are needed for the experimental measurement of $\mathscr{B} r\left(B_{s} \rightarrow \mu^{+} \mu^{-}\right)$. The result for the ratio of form factors, $f_{0}^{s}\left(m_{\pi}^{2}\right) / f_{0}^{d}\left(m_{K}^{2}\right)=1.046(44)(15)$, gives a value of the fragmentation fraction which agrees well with that of the $D_{s}^{+} \pi^{-} / D^{+} K^{-}$hadronic method and with LHCb's determination via a method employing semileptonic decays. This calculation included only a subset of the $B \rightarrow D l v$ data which is being analyzed by the FNAL/MILC collaboration for the determination of $\left|V_{c b}\right|$, so we can expect a considerable improvement when they finish analyzing their full data set.

\section{Outlook}

Lattice QCD calculations of non-perturbative parameters relevant for flavour phenomenology have achieved accuracies at the per-cent level for many key quantities. The agreement between results from different collaborations for the same quantities also allows an important check of lattice 
methods, especially in the light and charm sectors. In the bottom sector, the first $N_{f}=2+1$ results using a relativistic approach are very promising. For the next two years we expect new results from the FNAL/MILC, ETM, and RBC/UKQCD collaborations for decay constant, $B^{0}-\bar{B}^{0}$ mixing, and $B \rightarrow \pi l v$. One of the important steps in the improvement of lattice calculations expected in the next year is using simulations at the physical light quark masses. This would drastically reduce one of the main systematic, the one associated with the chiral extrapolation. There will be results with physical quark masses within a year from different collaborations: FNAL/MILC, BMW, RBC/UKQCD, and PAC-CS. The reduction of the dominant sources of errors will make necessary to include some uncertainties which were subdominant until now like isospin breaking, electromagnetic effects, or dynamical charm quarks.

\section{References}

[1] J. Laiho, E. Lunghi and R. S. Van de Water, Phys. Rev. D 81 (2010) 034503 [arXiv:0910.2928 [hep-ph]]. Updated information can be found in http://www.latticeaverages.org.

[2] M. Antonelli et al., Eur. Phys. J. C 69 (2010) 399 [arXiv:1005.2323 [hep-ph]].

[3] V. Cirigliano, J. Jenkins and M. Gonzalez-Alonso, Nucl. Phys. B 830 (2010) 95 [arXiv:0908.1754 [hep-ph]].

[4] A. Bazavov, et al., arXiv:1212.4993 [hep-lat].

[5] P. A. Boyle et al. [RBC-UKQCD Collaboration], Eur. Phys. J. C 69 (2010) 159 [arXiv:1004.0886 [hep-lat]]; V. Lubicz et al. [ETM Collaboration], Phys. Rev. D80 (2009) 111502. [arXiv:0906.4728 [hep-lat]];

[6] T. Bae et al., Phys. Rev. Lett. 109 (2012) 041601 [arXiv:1111.5698 [hep-lat]]; S. Durr et al., Phys. Lett. B 705 (2011) 477 [arXiv:1106.3230 [hep-lat]]; J. Laiho and R. S. Van de Water, PoS LATTICE 2011 (2011) 293 [arXiv:1112.4861 [hep-lat]]; RBC and UKQCD Collaborations, arXiv:1208.4412 [hep-lat].

[7] V. Bertone et al., arXiv:1207.1287 [hep-lat].

[8] A. Bazavov et al. [Fermilab Lattice and MILC Collaborations], PoS LATTICE 2012 (2012) 159 [arXiv:1210.8431 [hep-lat]].

[9] A. Bazavov et al. [Fermilab Lattice and MILC Collaboration], Phys. Rev. D 85 (2012) 114506 [arXiv:1112.3051 [hep-lat]].

[10] H. Na, C. T. H. Davies, E. Follana, G. P. Lepage and J. Shigemitsu, Phys. Rev. D 86 (2012) 054510 [arXiv:1206.4936 [hep-lat]].

[11] B. I. Eisenstein et al. [CLEO Collaboration], Phys. Rev. D 78 (2008) 052003 [arXiv:0806.2112 [hep-ex]].

[12] J. L. Rosner and S. Stone, arXiv:1201.2401 [hep-ex].

[13] BESIII Collaboration; talks presented at Charm 2012 and FPCP 2012.

[14] C. McNeile, C. T. H. Davies, E. Follana, K. Hornbostel and G. P. Lepage [HPQCD Collaboration], Phys. Rev. D 85 (2012) 031503 [arXiv:1110.4510 [hep-lat]]; H. Na, C. J. Monahan, C. T. H. Davies, R. Horgan, G. P. Lepage and J. Shigemitsu [HPQCD Collaboration], arXiv:1202.4914 [hep-lat]; A. Bazavov et al. [Fermilab Lattice and MILC Collaboration], Phys. Rev. D 85 (2012) 114506 [arXiv:1112.3051 [hep-lat]]. 
[15] J. P. Lees et al. [BABAR Collaboration], arXiv:1207.0698 [hep-ex].

[16] I. Adachi et al. [Belle Collaboration], arXiv:1208.4678 [hep-ex].

[17] J. A. Bailey et al., Phys. Rev. D 79 (2009) 054507 [arXiv:0811.3640 [hep-lat]].

[18] Y. Amhis et al. [Heavy Flavor Averaging Group Collaboration], arXiv:1207.1158 [hep-ex].

[19] C. Bernard et al., Phys. Rev. D 79 (2009) 014506 [arXiv:0808.2519 [hep-lat]]; J. A. Bailey et al. [Fermilab Lattice and MILC Collaboration], PoS LATTICE 2010 (2010) 311 [arXiv:1011.2166 [hep-lat]].

[20] S. -W. Qiu, C. DeTar, D. Du, A. S. Kronfeld, J. Laiho and R. Van de Water, PoS LATTICE 2012 (2012) 119 [arXiv:1211.2247 [hep-lat]].

[21] J. A. Bailey et al., Phys. Rev. Lett. 109 (2012) 071802 [arXiv:1206.4992 [hep-ph]].

[22] J. P. Lees et al. [BaBar Collaboration], arXiv:1205.5442 [hep-ex].

[23] Z. Liu, S. Meinel, A. Hart, R. R. Horgan, E. H. Muller and M. Wingate, arXiv:1101.2726 [hep-ph].

[24] R. Zhou et al., PoS LATTICE 2012 (2012) 120 [arXiv:1211.1390 [hep-lat]].

[25] H. Na, C. T. H. Davies, E. Follana, G. P. Lepage and J. Shigemitsu, Phys. Rev. D 82 (2010) 114506 [arXiv:1008.4562 [hep-lat]]; H. Na, C. T. H. Davies, E. Follana, J. Koponen, G. P. Lepage and J. Shigemitsu, Phys. Rev. D 84 (2011) 114505 [arXiv:1109.1501 [hep-lat]].

[26] C. Aubin et al. [Fermilab Lattice and MILC and HPQCD Collaborations], Phys. Rev. Lett. 94 (2005) 011601 [hep-ph/0408306].

[27] J. Beringer et al. [Particle Data Group Collaboration], Phys. Rev. D 86 (2012) 010001.

[28] J. Koponen, C. T. H. Davies and G. Donald, arXiv:1208.6242 [hep-lat].

[29] J. Laiho, E. Lunghi, R. Van De Water, PoS FPCP2010 (2010) 040 [arXiv:1102.3917 [hep-ph]];

[30] A. Lenz et al., Phys. Rev. D 83 (2011) 036004 [arXiv:1008.1593 [hep-ph]].

[31] A. Lenz et al., Phys. Rev. D 86, 033008 (2012) [arXiv:1203.0238 [hep-ph]].

[32] E. Gámiz et al. [HPQCD Collaboration], Phys. Rev. D 80 (2009) 014503 [arXiv:0902.1815 [hep-lat]].

[33] A. Bazavov et al., Phys. Rev. D 86 (2012) 034503 [arXiv:1205.7013 [hep-lat]].

[34] C. Albertus et al., Phys. Rev. D 82 (2010) 014505 [arXiv:1001.2023 [hep-lat]].

[35] C. M. Bouchard et al., PoS LATTICE 2011 (2011) 274 [arXiv:1112.5642 [hep-lat]].

[36] N. Carrasco et al., PoS LATTICE 2012 (2012) 104 [arXiv:1211.0568 [hep-lat]].

[37] A. J. Buras, Phys. Lett. B 566 (2003) 115 [hep-ph/0303060].

[38] K. De Bruyn, R. Fleischer, R. Knegjens, P. Koppenburg, M. Merk and N. Tuning, Phys. Rev. D 86 (2012) 014027 [arXiv:1204.1735 [hep-ph]]; K. De Bruyn, R. Fleischer, R. Knegjens, P. Koppenburg, M. Merk, A. Pellegrino and N. Tuning, Phys. Rev. Lett. 109 (2012) 041801 [arXiv:1204.1737 [hep-ph]].

[39] R. Aaij et al. [LHCb Collaboration], LHCb-CONF-2012-002.

[40] A. J. Buras, J. Girrbach, D. Guadagnoli and G. Isidori, Eur. Phys. J. C 72 (2012) 2172 [arXiv:1208.0934 [hep-ph]].

[41] RAaij et al. [LHCb Collaboration], arXiv:1211.2674 [Unknown].

[42] J. A. Bailey et al. Phys. Rev. D 85 (2012) 114502 [Erratum-ibid. D 86 (2012) 039904] [arXiv:1202.6346 [hep-lat]]. 